> electrodes. Electrochemists should develop more-effective electrolytes. Engineers need to develop tools for manufacturing these materials.

Government agencies and leading car manufacturers should fund this research, which will require billion-dollar investments. In our view, the best way to apportion this money is by targeting projects addressing key battery challenges, as the US Department of Energy's Advanced Research Projects Agency-Energy (ARPA-E) does. Developing batteries free from cobalt and nickel should be one priority.

As promising battery materials and cell technologies emerge, funding should be refocused towards improving their characteristics and viability. Production processes and costs must be addressed. We expect that synthesizing conversion electrode materials will require different steps, including forming certain nanoscale structures. Treatments involving series of solutions and gases, for example, might be borrowed from other sectors such as food, pharmaceuticals, filtration and composite manufacturing.

Lithium-ion battery manufacturers have already invested billions of dollars in dozens of 'giga-factories' to boost the electric-vehicle market. With co-ordination, thought and planning, these can be set on a new path to deliver the next generation of affordable batteries.

Kostiantyn Turcheniuk is a research scientist at the School of Materials Science \& Engineering; Dmitry Bondarev is an undergraduate student at the School of Chemical and Biomolecular Engineering; Vinod Singhal is a professor of business at the Scheller College of Business; and Gleb Yushin is a professor of materials science at the School of Materials Science \& Engineering, Georgia Institute of Technology, Atlanta, USA.

e-mail:gleb.yushin@mse.gatech.edu

1. Whoriskey, P. In Your Phone, In Their Air. The Washington Post (2 October 2016).

2. Amnesty International. Time to Recharge: Corporate Action and Inaction to Tackle Abuses in the Cobalt Supply Chain (Amnesty International, 2017).

3. Freeman, G. K.W. Process for recovery of cobalt by selective precipitation of cobalt-calcium double salt. US patent US6264904B1 (2001).

4. British Geological Survey. Cobalt Commodity Profile (BGS, 2009).

5. US Geological Survey. Mineral Commodity Summaries 2018 (USGS, 2018).

6. Brown, T. J. et al. World Mineral Production 2011-15 (British Geological Survey, 2017).

7. Nature 59,63-64 (1898).

8. Nitta, N., Wu, F., Lee, J. T. \& Yushin, G. Mater. Today 18, 252-264 (2015)

9. Wu, F. \& Yushin, G. Energy Environ. Sci. 10, 435-459 (2017).

10.Lee, J. et al. Nature 556, 185-190 (2018).

G.Y. declares competing financial interests; see go.nature.com/2ntpjme.

\title{
Pet genomics medicine runs wild
}

\section{Genetic testing for dogs is big business. It is too easy for companies to sell false hope, warn Lisa Moses, Steve Niemi and Elinor Karlsson. They call for regulation.}

\section{$\mathrm{L}$} ast year, a 13-year-old dog, let's call her Petunia, started having trouble walking and controlling her bladder and bowels. Distressed, her owners bought a US\$65 genetic test through a direct-toconsumer (DTC) company. It suggested that the pug carried a mutation that is linked to a neurodegenerative condition similar to the human disease amyotrophic lateral sclerosis (ALS, or motor neurone disease).

What published data there are ${ }^{1,2}$ suggest that as few as 1 in 100 dogs that test positive for this common mutation will develop the very rare disease, and Petunia's condition was also consistent with more-treatable spinal disorders. But her owners chose to put her to sleep, convinced that she would otherwise suffer progressive and irreversible paralysis and eventual death.

Genetic testing for pets is expanding. Hundreds of thousands of dogs have now been genetically screened, as Petunia was, and companies are beginning to offer tests for cats. But the science is lagging. Most of these tests are based on small, underpowered studies. Neither their accuracy nor their ability to predict health outcomes has been validated. Most vets don't know enough about the limitations of the studies, or about genetics in general, to be able to advise worried owners.

Pet genetics must be reined in. If not, some companies will continue to profit by selling potentially misleading and often inaccurate information; pets and their owners will suffer needlessly; and opportunities to improve pet health and even to leverage studies in dogs and cats to benefit human health might be lost. Ultimately, people will become more distrustful of science and medicine.

\section{WEAK SCIENCE}

Global spending on pets has grown by $14 \%$ over the past 5 years (see 'Animal lovers'), and worldwide annual spending on pet care is currently estimated to be about $\$ 109$ billion.
Genetics is one of the newest additions to this booming industry. Worldwide, at least 19 laboratories are now marketing genetic-testing products. Some vets use the results to help to diagnose sick pets, or to recommend that healthy ones be subject to expensive and sometimes invasive tests, such as bone-marrow biopsies. Some dog breeders use the tests to try to reduce the incidence of inherited diseases. At least one US veterinary hospital chain is now recommending genetic testing for all dogs, saying that the results allow "individualized healthcare" and can guide behavioural training.

We believe that three major problems plague pet genetic testing in its current state.

Lack of validation. In both humans and animals, mapping genetic variants to risk of disease is incredibly challenging. But most dog genetic tests are based on studies of candidate genes, which is a problematic approach. In such studies, researchers test a handful of human or animal genes for mutations that both match an expected inheritance pattern and seem likely to be pathogenic, for instance because they affect the structure of an expressed protein.

In humans, fewer than $2 \%$ of candidategene studies have stood $\mathrm{up}^{3}$ to further investigations using more-advanced methods, such as genome-wide association studies. As a result of these shortcomings, geneticists who study humans must now bring more evidence to designate a genetic variant as 'disease-causing. Through collaborative efforts involving industry, academia, physicians and patients, every clinical variant identified is now scored on a five-point scale, from 'pathogenic' to 'benign ${ }^{4,5}$. (Clinical variants are those that are linked to medically important phenotypes.)

No such careful reassessment has occurred in veterinary medicine. Many of the 200 tests offered by companies are based on only a single small candidate-gene study (see, for example, go.nature.com/2nquntx). 


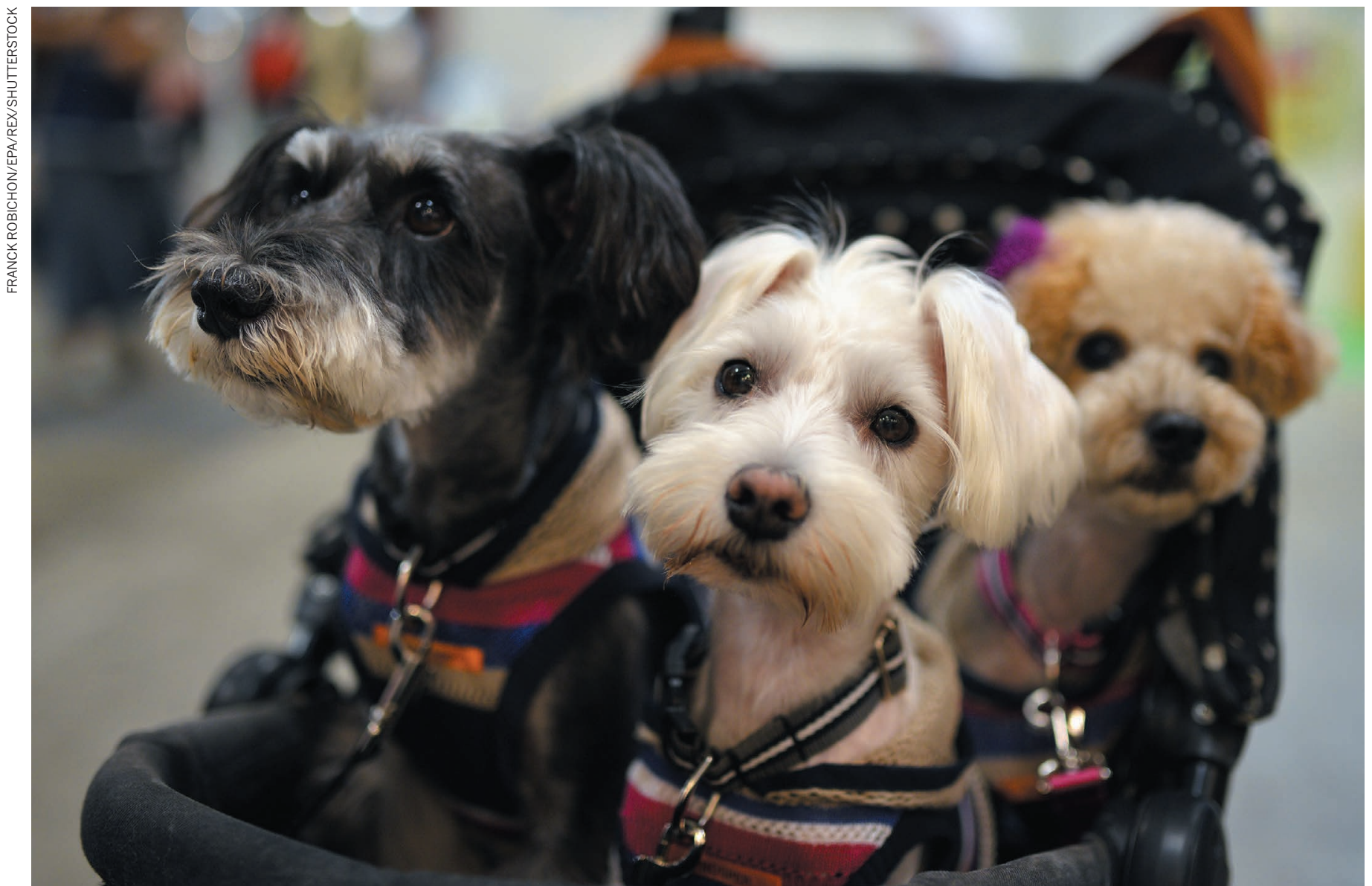

Spending on pet care is increasing around the world.

The data obtained are rarely made public. Neither is the information required to contextualize the results. Providers who have followed up with owners to check the accuracy of their tests' health predictions have reported case studies involving a small number of $\operatorname{dogs}{ }^{6}$, but nothing on the scale needed to provide statistically reliable results ${ }^{7}$.

Imprecise results or interpretation. A recent analysis of DTC results supplied by human genetic-testing companies found that, of all genetic variants presented in people's test results, $40 \%$ were inaccurate ${ }^{8}$. Other variants were reported by DTC companies to increase disease risk, but are considered benign by independent labs. Finally, the companies failed to screen for all known disease-linked mutations for any given gene, giving an incomplete picture of genetic susceptibility to disease.

The same problems apply to the DTC products now offered for pets. Dogs, for example, are frequently screened for mutations in the gene $A B C B 1$ that are associated with dangerous sensitivity to several common drugs. Three different mutations have been associated with this phenotype, but the documentation provided by genetictesting companies implies that they test for only one. Thus, a dog declared 'clear' for a given gene might still harbour other known, clinically relevant mutations in that gene that the company has not tested for.

Conflicts of interest. In the absence of effective industry self-policing or government regulations, there are many potential conflicts of interest associated with making profits from pet owners. As an example, a pet health-care corporate database could be used to identify the breed of an owner's dog. The corporation owning the database might then notify the clinic (which might be owned by the same company) that a genetic test for a specific illness is warranted, regardless of that test's medical value. If the test comes back positive, the clinic's vet might recommend preventive steps, such as specific pet foods (made by the same company), periodic screening tests (performed by the company's clinical lab), and more-frequent exams (performed at the company's vet clinics), even though there may be low or no risk of disease in the first place.

\section{TSUNAMI OF DATA}

The companies do provide multiple caveats about interpreting genetic test results in their terms and conditions. Yet their warnings are not as prominent as their claims. And companies tend to be vague about which variant they test for, even in publications ${ }^{6}$.

All of this is worrying because veterinary medicine is about to be hit by a tsunami of genomic data. Currently, pet owners can purchase the tests directly from companies and obtain results that ostensibly report on risk for more than 100 different diseases for less than $\$ 200$, and some kits provide a 'health report' to take to the vet. Within the next five years, owners will have the option of whole-genome sequencing for pets of any species, using the same technology used for human medicine. Such results could accelerate genetic-based diagnoses and even the genetic editing of pets.

In principle - with large, well-powered studies, involving perhaps tens of thousands of animals - genetic testing could be used to predict the risk of common diseases affecting millions of pets, including cancer, heart disease, diabetes and epilepsy. The use of pets in preclinical and clinical research to benefit humans is growing. For certain diseases, such as cancer and diabetes, researchers are sometimes using pet dogs as models in preference to lab rodents. Although it is harder to control environmental variables and to obtain high numbers of subjects, the use of dogs offers the advantages of spontaneous disease models that might be closer to the human version. Additionally, pet owners voluntarily enrol their pets and continue to feed and care for them at home. Thus, many of the costs and regulations that govern the 


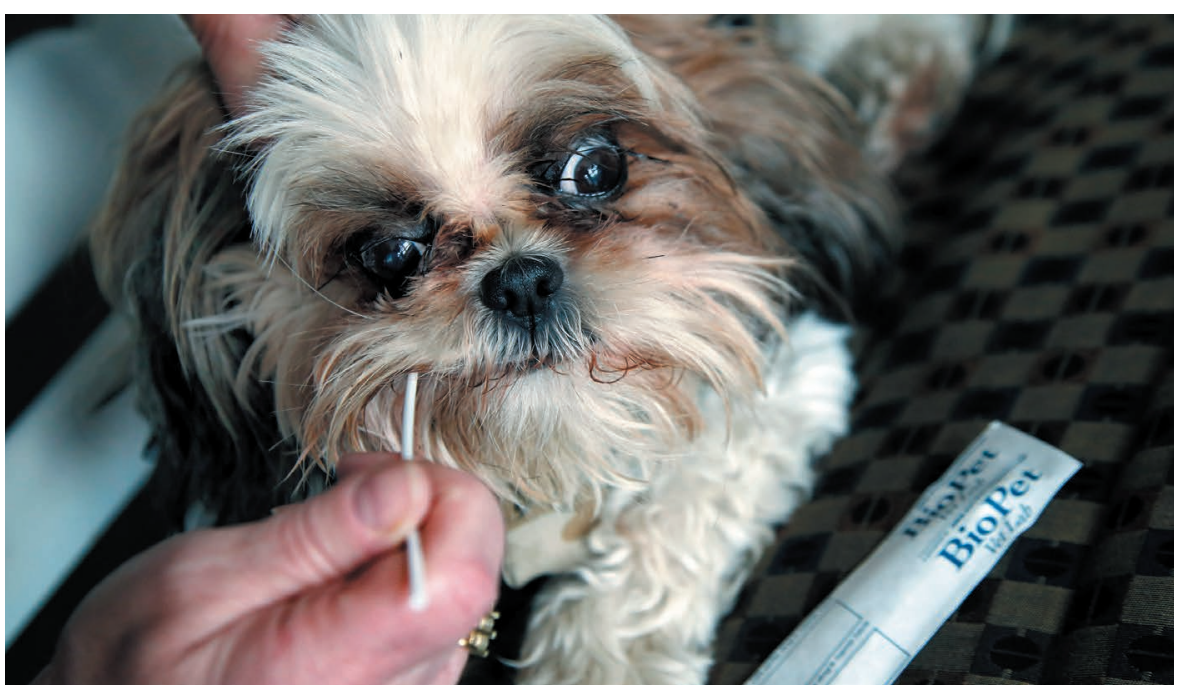

A cheek swab is all that's needed for a genetic test.

same species when they are used in the lab can be bypassed. Such methodological shifts make it imperative that the science of pet genetics is robust.

Largely because current regulatory mechanisms for pets focus on treatments, not diagnostics, there is no oversight of genetic testing for pets in the United States or in the European Union. To our knowledge, neither the US Food and Drug Administration (FDA) nor the European Medicines Agency (EMA) has proposed quality standards, or sought public input about the tests' pros and cons.

To bring the untamed wilderness of pet genetic testing under control, we propose five steps.

Establish standards. All stakeholders, from pet owners and the companies providing the tests to researchers and regulators, should work together to establish standards both for testing methodology and for the reporting of test results. Samples should be collected, stored, shipped and analysed in specified ways; the number of animals should exceed a certain threshold to support claims made about a specific test; and reports should meet specified criteria. Reports must be sufficiently clear, with information about potential caveats provided at a predetermined point in the document and in easy-to-read language. Organizations such as the World Small Animal Veterinary Association could take the lead on convening meetings with this goal.

Create guidelines. A select group of stakeholders - which would comprise working groups - could develop guidelines that specify the standards that should be adopted. Such a document could be similar to the ethics guidelines ${ }^{9}$ for the use of novel technologies in lab-animal research developed by the British Small Animal Veterinary Association science subcommittee. Ideally, the instructions would have global reach, and as pet genetic testing grows, such guidelines might eventually need to become law.

Share data. Existing pet genetic databases, which are produced by industry, academia and government agencies, should be shared among all parties, with steps taken to ensure that individual pets and owners can't be identified. Companies might be incentivized to share data if the proposed guidelines require sample sizes that can be achieved only through pooling data. To improve transparency, stakeholders can draw on the knowledge accumulated across the past two decades by scientists researching human genetics, who have been grappling with issues around data sharing and patient privacy.

Recruit tools and expertise. Bioinformaticians, computer scientists trained in machine learning, and others with expertise in big data will be needed to manage and analyse the tremendous volume of incoming information. Such specialists might be persuaded to enter the burgeoning field of pet

\section{ANIMAL LOVERS}

In the United States and Europe, spending on pet care has steadily grown over the past six years.

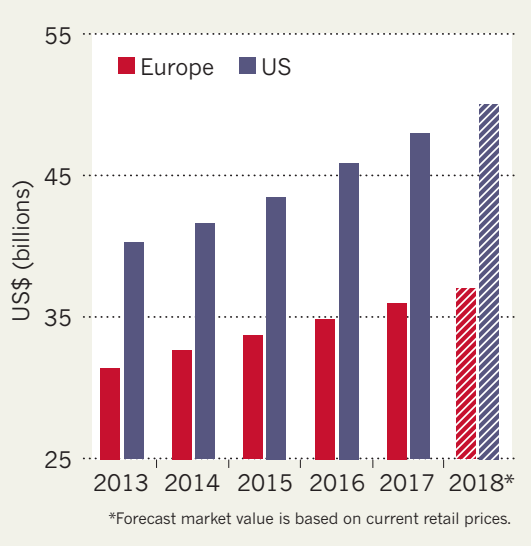

genetics because of the potential financial gains. Or they could be lured by the possibility of pet genetics offering an inroad into solutions to human disease.

Educate counsellors. A cadre of pet genetic counsellors needs to be established from among vets and others working in animal care. These professionals would provide support and advice to pet owners following genetic tests and could potentially be affiliated with their counterparts in human genetic counselling at leading academic medical centres.

\section{DATA ETHICS}

Other issues might require ongoing public dialogue and the appointment of expert advisory boards. For instance, should for-profit pet-testing firms have unfettered access to all the results they generate, for internal corporate use or to sell to other companies engaged in pet care - as is the case now? Can an owner, who might want to breed their pet and sell the offspring, keep any results secret (as they can currently) or should they be required to divulge this information to buyers?

In the United States alone, some $70 \%$ of households own pets. Done right, the use of genetic testing in companion animals could be a powerful way to better connect people to the possibilities of genetics for treating disease. Done wrong, it could erode trust in science for an increasingly sceptical public..

Lisa Moses is a research scholar at the Center for Bioethics, Harvard Medical School, Boston, Massachusetts, USA; and a practising veterinarian in the Pain and Palliative Care Service at the MSPCA-Angell Animal Medical Center, Boston, Massachusetts. Steve Niemi is a veterinarian and director of the Office of Animal Resources, Harvard University, Cambridge, Massachusetts. Elinor Karlsson is assistant professor at the University of Massachusetts Medical School, Worcester, Massachusetts; and director of vertebrate genomics at the Broad Institute of Harvard and MIT, Cambridge, Massachusetts. e-mails:lisa_moses@hms.harvard.edu; sniemi@fas.harvard.edu; elinor.karlsson@ umassmed.edu

1. Coates, J. R. \& Wininger, F. A. Vet. Clin. North Am. Small Anim. Pract. 40, 929-950 (2010)

2. Zeng, R. et al. J. Vet. Intern. Med. 28, 515-521 (2014).

3. Ioannidis, J. P. A., Tarone, R. \& McLaughlin, J. K. Epidemiology 22, 450-456 (2011)

4. Richards, S. et al. Genet. Med. 17, 405-424 (2015).

5. Harrison, S. M. et al. Genet. Med. 19, 1096-1104 (2017).

6. Donner, J. et al. PLoS Genet. 14, e1007361 (2018)

7. Goldstein, B. A., Navar, A. M., Pencina, M. J. \& loannidis, J. P. A. J. Am. Med. Inform. Assoc. 24, 198-208 (2017).

8. Tandy-Connor, S. et al. Genet. Med. https://doi. org/10.1038/gim.2018.38 (2018).

9. Yeates, J. W. J. Small Anim. Pract. 57, 67-73 (2016).

E.K. declares competing interests; see go.nature. com/2mwyv2b. 